This item was submitted to Loughborough's Research Repository by the author.

Items in Figshare are protected by copyright, with all rights reserved, unless otherwise indicated.

\title{
RFID based automatic speed limit warning system
}

PLEASE CITE THE PUBLISHED VERSION

http://ukacc.group.shef.ac.uk/?page_id=87

PUBLISHER

UKACC

VERSION

AM (Accepted Manuscript)

LICENCE

CC BY-NC-ND 4.0

REPOSITORY RECORD

Yang, Huanjia, and Shuang-Hua Yang. 2019. "RFID Based Automatic Speed Limit Warning System”. figshare. https://hdl.handle.net/2134/11874. 
This item was submitted to Loughborough's Institutional Repository (https://dspace.lboro.ac.uk/) by the author and is made available under the following Creative Commons Licence conditions.

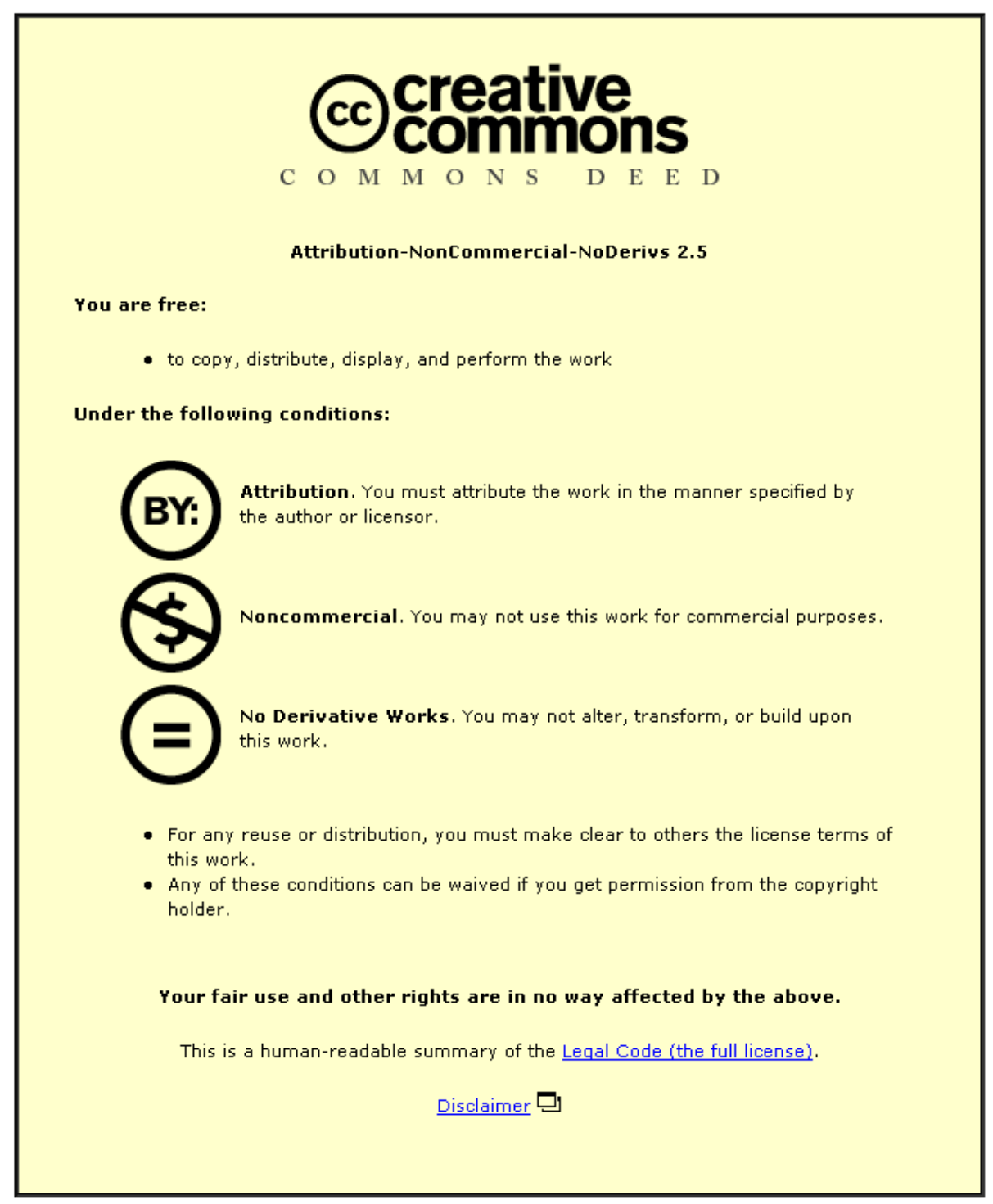

For the full text of this licence, please go to: http://creativecommons.org/licenses/by-nc-nd/2.5/ 


\title{
RFID Based Automatic Speed Limit Warning System
}

\author{
Huanjia Yang, Shuang-Hua Yang \\ Department of computer Science, Loughborough University, Loughborough, LE11 $3 T U$ \\ UK (Tel: +44-1509-635648; e-mail: \{H.Yang, S.H.Yang\}@lboro.ac.uk).
}

\begin{abstract}
Speeding is one of the major causes of road accidents. As drivers usually claim they exceed the speed limit unintentionally, having an in-vehicle automatic speed limit warning system could help to prevent a significant number of speeding and accidents from happening. Existing systems cannot provide consistent and satisfactory performance. In this paper we investigated automatic speed limit transmission based on RFID technologies. We conclude that both passive and active RFID systems show potential in such scenario, but are suitable for different scales of applications. For both technologies, challenges are identified and addressed, with system prototypes proposed and demonstration systems developed to prove the feasibility of our design concept.
\end{abstract}

Keywords: RFID, speed limit, driver assistant system

\section{INTRODUCTION}

According to research by US Department of Transportation, speeding is a factor in about one-third of all fatal crashes, killing more than 1,000 people in the country every month. In 2006, more than 13,500 people died in speed-related crashes. The National Highway Traffic Safety Administration estimates that the economic cost of speed-related crashes is more than $\$ 40$ billion each year (DOT, 2009). Significant research has been conducted on the effect of the speed of individual vehicles involved in the crash, showing an exponential relation between speed and crash rate (Kloeden et al., 2001). In addition, vehicles travelling at $80 \mathrm{mph}$ use 10 $20 \%$ more fuel than those travelling at $70 \mathrm{mph}$. There is no doubt that an increase in vehicle speed increases the emissions of carbon dioxide, nitrogen oxide and generates more noise (Department for Transport, 2000).

Although more than $80 \%$ of the European drivers state that driving too fast is a contributory factor in road accidents (Cauzard et al., 2004), many drivers still claim that they exceed the posted speed limits unintentionally. One reason could be that the driver is unaware of the speed limit. It may be assumed that this is an unintentional violation. This is either because a speed limit sign was absent or because the driver missed seeing it. In both cases the road characteristics are insufficiently informative about the speed limit in force. Another reason for unintentional exceeding of speed limit is underestimation. All motor vehicles have a speedometer to objectively check the driving speed. Nevertheless many drivers rely on their subjective perception or 'feeling' of their speed when it comes to determining their speed. However, human perceptual skills and limitations affect the subjective experience of speed and may lead to an underestimation of the true speed. This underestimation can easily occur in a situation where a high speed has been maintained over a long period or where drivers must reduce their speed significantly after a period of high speed driving and where there is little peripheral visual information. Thus, reading road speed limits automatically and alerting the drivers when they exceed the speed limit are potentially useful as they can help to control speeding and thus prevent a significant number of accidents from happening. The remainder of this paper is organized as follows: first we briefly introduce existing speed limit systems and their features; Then we introduce our approach beginning with passive RFID technology, with antenna direction and tag response speed been studied in detail to prove the feasibility of the design, Then the use of active RFID technology is investigated and we propose solutions to prevent active tag confusion. Finally a test system is developed, to demonstrate the feasibility of our design.

\section{EXISTING SYSTEMS}

The current speed warning systems include GPS systems and speed sign recognition based on real-time image processing. GPS based speed warning systems are usually integrated with GPS navigation systems. A GPS sensor receives satellites signal and calculate the coordinators of the vehicle. The system then locates the vehicle's position on pre-loaded road map and indicates the corresponding speed limit according to a speed limits database. Such system suffers from the following shortages: 1). the map and the speed limit database are pre-loaded into the device, which means they are not updated in real-time and could contain out of date information. Temporary speed limits, the changing of the road layout and speed signs may cause inconsistency between the device readings and the real world speed limit. 2). GPS localization has a limited tracking accuracy of 10 to 20 metres. GPS navigation systems use various calibration algorithms, but under certain circumstances such as in downtown environment they are still not able to ensure that the position of vehicle is correctly located, which may lead to an incorrect speed limit reading. 3). GPS systems rely on the satellite signal which are not reliable, especially during bad 
weather and the satellites are not even accessible while driving through tunnels or a high-rise district.

Speed sign recognition (Kohashi et al., 2004; Makanae, 2004) is a technique based on real-time image processing. Cameras are installed on the front of the vehicle and continuously capture images ahead of the vehicle. Algorithms are developed to identify and read the speed limit signs on the road by processing the images so captured. Such systems have difficulty working in bad weather with decreased visibility, and are not able to provide any readings when the road signs are missing or obscured by other vehicles passing or overgrow vegetation.

\section{RESEARCH AND DEVELOPMENT METHOD}

RFID is an Auto-ID technology using radio waves to automatically identify people or objects. The basic components of a typical RFID system include: the transponder or the tag, which is a microchip in which a unique serial code is stored and transmitted when necessary via an attached antenna; the RFID reader, which is used to receive and identify the information sent by tags. RFID is already in use in some the transport related applications, such as vehicle parking or detection systems, where tags are attached to vehicles and readers are deployed at control points to identify the vehicles passing by.

The idea of our approach is to adopt RFID to help drivers gather speed limit data on the road; to alert the driver when speeding occurs; to limit the vehicle speed automatically and to record driver's behaviour when necessary. However, since it is the roadside devices that are providing the information, we are interested in using RFID in the opposite way to current systems by locating tags at roadside and readers in vehicles. In our research the speed limit are stored in RFID tags which will be embedded on signposts or certain points of road surface. When a vehicle passes such a signpost or surface point on a specific road, a reader installed in the vehicle will retrieve the corresponding speed limit information from the tags. Such information could then be indicated on an in-car display, or be used for a driver warning system to alert the driver they are speeding. As RFID provide us a non-contact data transfer between the tag and the interrogator without the need of line-of-sight data gathering, the speed limit readings of our system would be reliable in all weather conditions. Without the need to listen to satellites or base stations, the system will be suitable for all areas including tunnels and downtown areas. New road plan and temporary speed limit can be easily introduced by changing or implementing new tags. Because the in-car devices do not rely on any pre-loaded map or database, it will be simpler, cheaper and easier to maintain.

\section{PASSIVE RFID BASED SYSTEM}

In passive RFID the focus is on the location of tags. Tags may be placed on the existing signposts directly, mounted on overhead stands or on the road surface and the reader antenna may be placed on top or underneath the vehicle or on the chassis pointing straight down.

\subsection{Implementing passive tags on existing signpost}

The idea of placing the passive tags on the existing signposts is quite simple. It is easier and requires no further road sign planning. But such implementations are not straightforward.

Antenna direction: passive RFID reader antennas are directional. This means tag can only be read when the reader antenna is directly facing it and it is parallel to the antenna. These restrictions make tagging existing signpost difficult.

It is preferable to deploy all passive tags on those signposts at the left road side. This is because of the following:

Firstly, existing signposts have been deployed at various places on road as shown in Fig. 1. They can be at the left road or right roadside, on overhead structures or in the division between carriageways. In order to read the tags attached to these signpost, a RFID reader needs not only to have sufficient read range, which might possibly be achieved by newly designed reader chips, but also needs to point its antenna right towards them. To do this the reader would need to either move its antenna toward each signpost, or have multiple antennas that cover all possible directions. Neither of these seems to be practical especially when our system is developed as a third party add-on for vehicles.

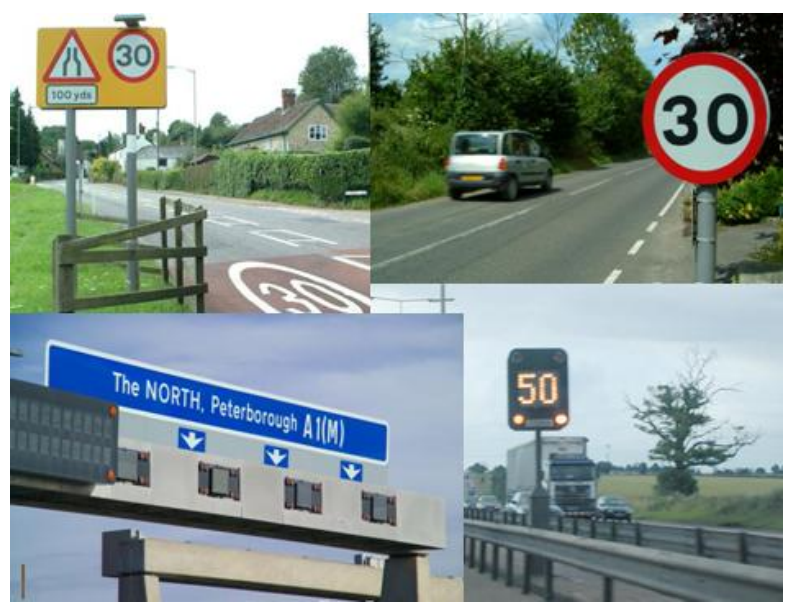

Fig.1. Speed signs in UK at various relative directions

Secondly, even if both of the reader antenna implementations described in the last paragraph are considered to be possible, the speed tags deployed for the opposite carriageway, which may have a different speed limit, may be read as ghost tags by the multi-antenna readers with antennas pointing in all directions or by the single-antenna reader when it points its antenna towards the signposts between the carriageways. To avoid such confusion the tags should always be geographically separated, and the best way to do so is to deploy the tag only on the left hand side of the road so that the antennas on the vehicles in both carriage ways point in completely different directions and will not read the tags on the opposite side.

For these two reasons all the signposts with passive tags must be deployed on the left roadside. This may require additional 
road sign deployment as existing signposts are not always deployed on the left roadside. However, to make the tag-onsignpost solution feasible there are two more problems to be overcome which are currently unsolved.

Read range. We have tested passive RFID systems using the UHF band, which have the longest reading range. These tests are carried out in Holywell Park, Loughborough University with various readers and tags and show a furthest tag reading distance of $6 \mathrm{~m}$, this is insufficient for road application. According to UK Highway Agency documentation, as shown in Fig. 2, the standard lane width for motorway and dual carriage way is $3.65 \mathrm{~m}$. The ability to read EPC class $1 \mathrm{Gen} 2$ tag at up to 6 meters may be enough for vehicles on dual carriage ways or for those using lane 1 and 2 on motorways. But in reality there is also considerable distance between the signpost and the side of lane 1 due to safety reasons, and if there is a cycle lane in-between them, this will further increase this distance. Thus we must conclude that only vehicles using lane 1 may have a reasonable tag reading performance. This analysis is based on the latest UHF passive RFID products; the read range of the UHF reader might be increased further in the future, but at the moment, no appropriate hardware is available in the market.

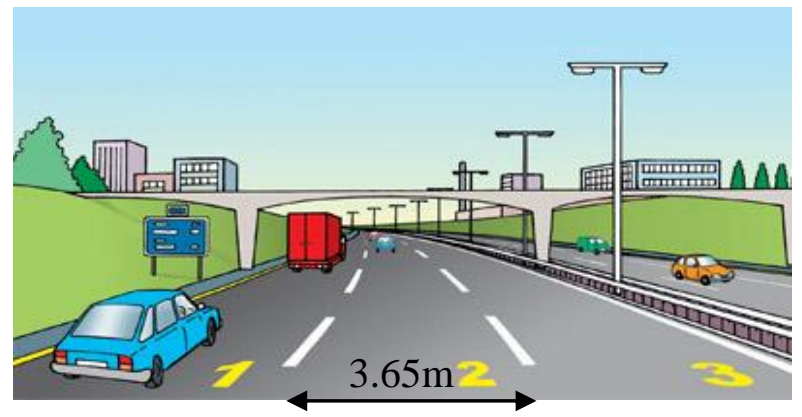

Fig.2. UK highway lane number and width

On road obstacle: For multi-lane carriageways the reader on a vehicle in the outside lane will be blocked by another vehicle in a slower lane that lies between it and the signpost. The situation becomes even worse when we take into account the fact that that the slower lanes are often used by large vehicles such as HGVs. As the UHF passive RFID reading operation is very sensitive to obstacles between the tag and antenna, it is very likely that the speed tag reading will fail when obstacle vehicle are present. The only way to avoid this is to deploy tags somewhere either overhead or underneath the vehicles. But neither of these two solutions would now comply with the tag-on-signpost approach.
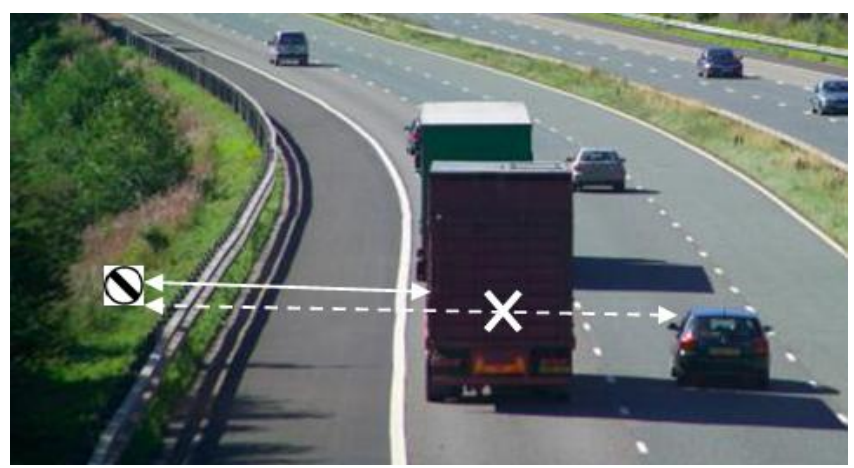

Fig.3. Reader on a vehicle blocked by HGV in slower lane

\subsection{Implementing passive tags overhead}

Placing tags overhead is one of the two solutions that avoid both reading confusion and on-road obstacles. Fig.4 shows such a system that has been implemented in Florida, USA for highway tolling and traffic control. The difference is that the readers are implemented overhead and tags are deployed in vehicle under the windshield. And although the system is advertised as a UHF passive RFID system, the tag actually has batteries installed inside to enable it to interact with the driver when their prepaid account does not contain enough fund for paying the toll. The problem with this solution is the implementation cost, especially if considering a system that can potentially be implemented on all roads, As most of the roads do not have overhead sign stand, building such stands for all current roads seems to be impractical.

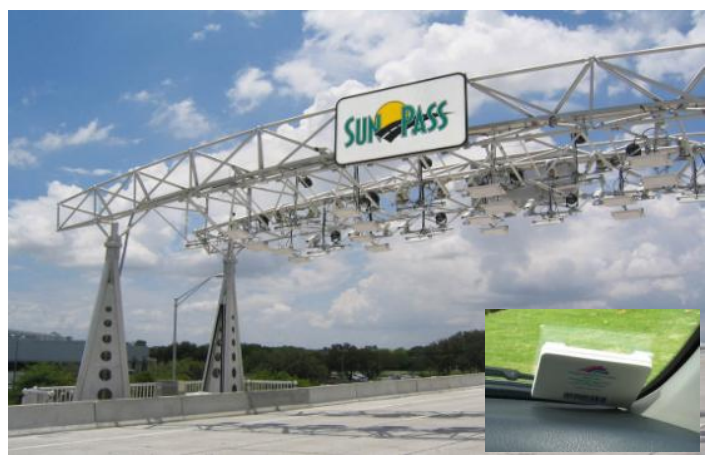

Fig.4. SunPass RFID Tolling and Traffic Control System

\subsection{Implementing passive tags on road surface}

Placing passive tags on the road surface, therefore, is the last option available. This is similar to the overhead tag solution, in that placing passive RFID tags on the road surface does not have the obstacle problem. In addition, the read range required is even shorter and more controllable then with the previous solutions. This is thus our preferred design and we will discuss how the following issue can be addressed;

Reading speed / Tag response time: In a static passive RFID system where the reader and tags are fixed or have a slow relative movement speed, the shorter the distance between the reader and tag, the better reading we generally can achieve. However, in our project, shorter distance doesn't always mean better performance. Because the vehicles may be travelling at very high speed and as the distance between the tag and reader antenna is only $30-50 \mathrm{~cm}$, the tag on the road surface may be within the reader antenna's effective reading area for only a very short time. This means we are challenging the limit of passive RFID reading speed, or in other words tag response time.

The system shown in Fig. 5 has been tested by researchers from Miyagi University in Japan (Yoshimichi, 2006). Passive HF RFID tags are attached on the road surface inside the university campus and a reader is installed in a vehicle with its antenna mounted at the end of the car. The researchers 
used HF RFID reader and tags in the design. Because of the slow speed of the HF RFID the experiment result shows that the system can only read tags correctly at vehicle speed up to $20 \mathrm{~km} / \mathrm{h}(12.5 \mathrm{mile} / \mathrm{h})$, which is really very practical.

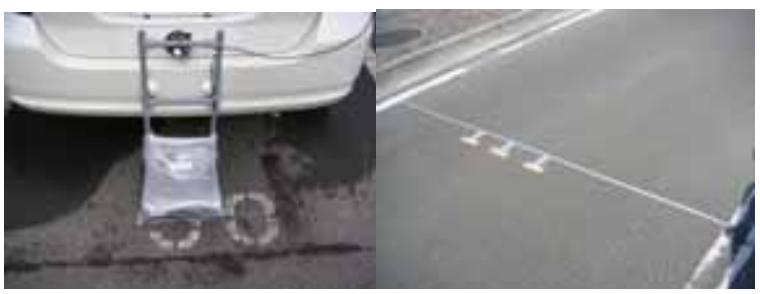

Fig.5. In-vehicle Signing System (Yoshimichi, 2006)

UHF passive RFID systems have a much faster reading speed than the other RFID systems in the lower frequency bands. Thus we carried out a similar field trial using the Skyetek M9 UHF passive RFID embedded module. The test results are shown in Fig. 6, which shows a maximum moving speed of $25 \mathrm{mph}$ for the reader to successfully read a passing tag. This speed is however is still insufficient for our application.

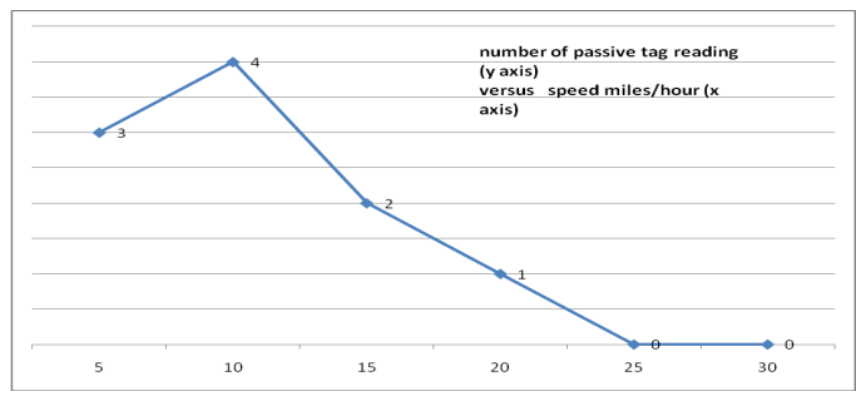

Fig.6. In vehicle test with Skyetek M9, tags at road side

To understand this result, we have identified the key performance parameter that relates to the maximum moving speed is the reader's tag response time. Tests have been carried out on the Skyetek M9 with tools from the manufacturer and show an average tag response time of $35 \mathrm{~ms}$ These tests also show that the M9 antenna effective area is around $40 \mathrm{~cm}$ long at a tag-reader distance of $50 \mathrm{~cm}$. Thus the maximum relative speed between tag and reader for a correct reading to be obtained can be given as:

$$
\frac{3600 \mathrm{~s} / \mathrm{h}}{0.035 \mathrm{~s}} \times 0.4 \mathrm{~m} \times \frac{1}{1000 \mathrm{~m} / \mathrm{km}} \approx 41 \mathrm{~km} / \mathrm{h} \approx 25 \mathrm{mile} / \mathrm{h}
$$

This result closely matches the M9 in-car field test result presented in Fig.6, which showed a maximum speed of $25 \mathrm{mph}$ that is insufficient for our project. But we have identified a feature of the EPC Class 1 protocol called "ScrollAllID" or "global scroll", which is not supported by the M9 device, which can help to achieve the expected reading performance in our design.

Some passive readers implement this feature of the EPC Class 1 protocol called "ScrollAllID," or "GlobalScroll." This feature allows the reader to bypass any collision detection and resolution algorithms, and can consequently yield read rates of over 400-500 tags/sec (Ramakrishnan, 2006). Since the GlobalScroll mode avoids collision detection, it is generally not recommended except in very well-controlled conditions. However, when placing tags on road surface the reader-tag distance, tag-antenna relative direction and the number of tag within the antenna reading area can all be easily controlled, thus we can expect this feature to be fully working and providing the expecting performance.

Read rates of over 400 tags/sec means approximately $2.5 \mathrm{~ms}$ response time from the passive RFID tag, considering a vehicle travelling at $100 \mathrm{mph}$, it moves about 45 metres per second. The chassis height of a typical salon, hatchback or van is about $20-30 \mathrm{~cm}$ from the road surface and according to the lab tests the effective range of the reader antenna at this distance is about $40-50 \mathrm{~cm}$ long. The reader can thus have 9$11 \mathrm{~ms}$ over the passive tag and thus should, in theory, be able to read the tag successfully. Actually, if we use the same equation as we used to calculate the maximum reading speed of the M9, we can calculate the theoretical maximum moving speed for a reader that supports GlobalScroll to read a tag as

$$
\frac{3600 \mathrm{~s} / \mathrm{h}}{0.0025 \mathrm{~s}} \times 0.4 \mathrm{~m} \times \frac{1}{1000 \mathrm{~m} / \mathrm{km}} \approx 576 \mathrm{~km} / \mathrm{h} \approx 358 \mathrm{mile} / \mathrm{h}
$$

One of the passive UHF RFID readers that supports this feature is the Alien 8800 reader; it is also claimed to be the fastest UHF reader in the market. Researchers in India have tested an Automatic Vehicle Identification (AVI) system based on the Alien 8800 reader (Butani et al., 2007). Although the system design and purpose are not the same as our project, it did prove that the particular reader-tag combination they were using can correctly read tag information at a relative movement speed of $150 \mathrm{~km} / \mathrm{h}$ (approx. $93 \mathrm{mph}$ ) between the reader and the tag.

As the Alien 8800 reader claimed to be the fastest reader in the market, we tested such a reader in our lab environment. However, in our own test of the Alien 8800 it did not reach the full theoretical reading speed suggested by GlobalScroll and shows an average tag reading speed of $7 \mathrm{~ms}$ to $8 \mathrm{~ms}$. With this test result and the previous equation the maximum moving speed for Alien 8800 to read a passing tag is:

$$
\frac{3600 \mathrm{~s} / \mathrm{h}}{0.0075 \mathrm{~s}} \times 0.4 \mathrm{~m} \times \frac{1}{1000 \mathrm{~m} / \mathrm{km}} \approx 192 \mathrm{~km} / \mathrm{h} \approx 120 \mathrm{mile} / \mathrm{h}
$$

This is very close to the result of previous AVI system field test, and is more than sufficient for our application.

\section{ACTIVE RFID BASED SYSTEM}

Passive RFID technology shows great potential in large-scale applications where a massive speed tag implementation is required. On the other hand, for small-scale applications, passive RFID Readers are still too expensive and consume considerable amount of power while working, while their hardware size brings difficulties with vehicle integration. For simpler scenarios like temporary speed limit warning, a simpler system that acts as an add-on to existing systems could be more practical. Active RFID is considered to be suitable in this case. With slightly higher roadside tag cost and a limited but acceptable battery life, it allows the reader 
to be a much simpler, cheaper and smaller device. This enables possible integration with existing systems such as GPS navigators. Active RFID have longer read range and more reliable tag reading to facilitate the tag implementation. Instead of putting tags on road surface, road workers could simply put the speed tags on the temporary traffic signs.

The issue raised when using active RFID for speed limit transmission is tag confusion. Tag confusion happens because the RF range of active transmitter cannot be accurately controlled. The RF range of different active speed tags overlap and will confuse the in vehicle assistant system when multiple tags are read at the same time. Tag overlapping may happen on all roads because the two carriageways in opposite directions may have different speed limits. As shown in Fig.7, overlapping will also happen with multilayer roads and at crossroads.

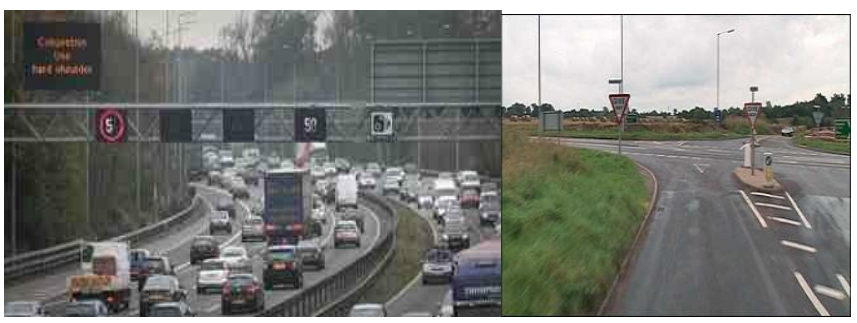

Fig.7. Active tag confusion

We have evaluated two different ways to address the tag confusion issue, which are directional antenna and traffic direction identification.

\subsection{Directional antenna}

The first solution is to use directional antenna to avoid tag confusion. A directional antenna or beam antenna is an antenna which radiates greater power in one or more directions allowing for increased performance while transmitting and receiving and reduced interference from unwanted sources. By adopting directional antenna on active speed tags, we could focus the speed limit signal transmission in a particular direction and let it cover only the area where the speed limit is valid.

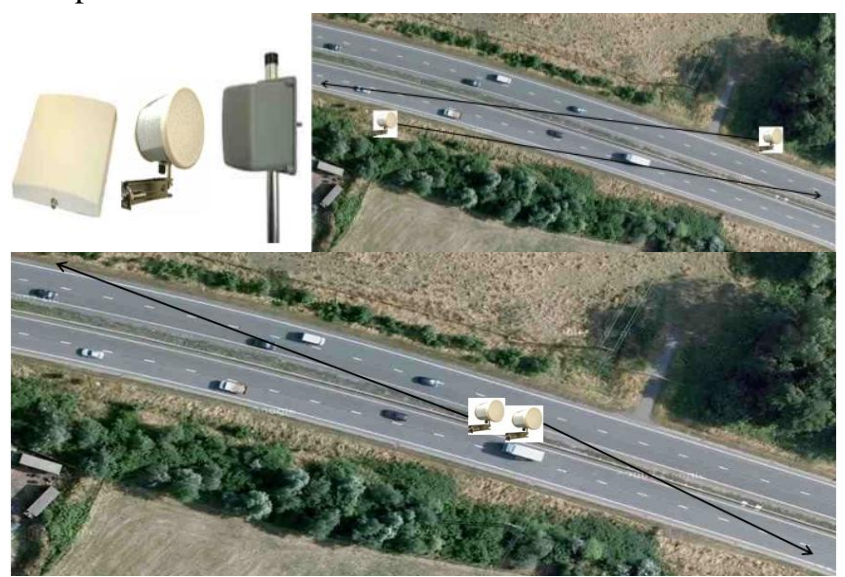

Fig.8. Directional antenna and their locations

The speed tags with directional antenna must be deployed between the carriageways, pointing towards the opposite directions to avoid any possible confusion, while at the same time covering the specific carriage to which it needs to broadcast the speed limit information. (Fig. 8)

We test our design with Jennic JN5139 wireless controller and three different types of $2.4 \mathrm{GHz}$ outdoor directional antennas having an effective angle of 30,45 and 60 degrees respectively. In our tests with these directional antennas, however, the RF field that the tag covers shows an imperfect directionality. For example, the area that was covered by a 30 degree $12 \mathrm{~dB}$ directional antenna is shown in Fig.9 Although a much longer distance on the main direction is achieved, the tag also covers a considerable large area outside the main 30 degree transmission angle, thus the receivers picked up the speed limit signal consistently even behind the antenna. This is because the directionality of the antenna is only a relative feature. According to the directional antenna gain chart shown in Fig.9, a directional antenna only assures a much higher antenna gain in the main direction, but at the same time the RF power transmitted in the other directions at a closer distance is not negligible. Due to this reason the directional antenna itself cannot fully address the tag confusion issue. RF shielding is a possible way to improve the directionality of an antenna in certain circumstances. However, our test results with various RF shielding materials shows that shielding still cannot provide fully satisfactory performance due to the very short distance between the antenna and the opposite carriage way. (Fig. 9)

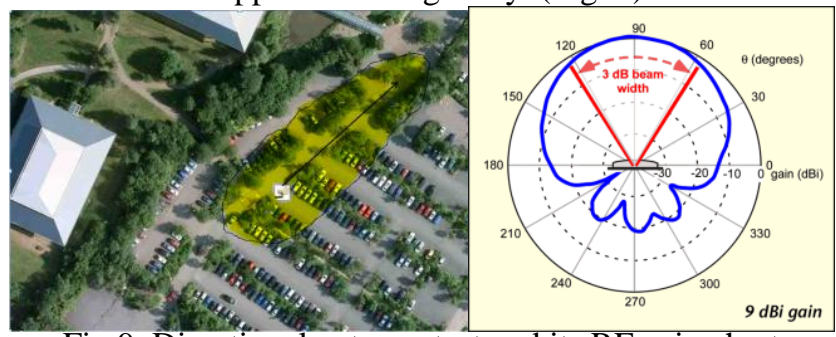

Fig.9. Directional antenna test and its RF gain chart

\subsection{Traffic direction identification - Bearing measurement}

As a directional antenna by itself fails to address the tag confusion issue, we have thus designed an improved solution that integrates an electronic module to measure the device's bearing. These modules are solid-state electronic devices built out of two or three magnetic field sensors and provide data for an on-board microprocessor. The correct bearing relative to the device is calculated using trigonometry.

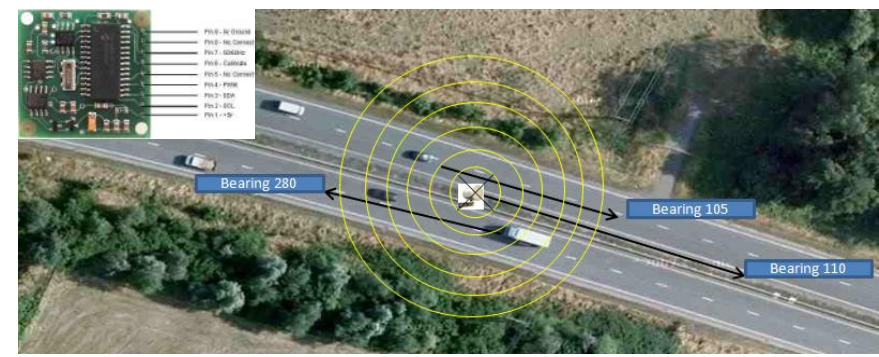

Fig.10. Design with bearing measurement module

The bearing measurement modules are integrated with both active speed tags and receivers. By placing the speed tags with the module board pointing in the direction of the 
corresponding carriageway, the module provides the tag processor with the carriage way bearing (CB). The $\mathrm{CB}$ is sent together with the speed limit information in via tag broadcasting. A receiver in the vehicles retrieves both the speed limit and the $\mathrm{CB}$, and determines from its own bearing measurement module the vehicle heading $(\mathrm{VH})$. It then compares the value of $\mathrm{CB}$ and $\mathrm{VH}$ to determine whether the received speed limit is valid for the vehicle.

This direction identification solution avoids the high cost and the bulky size of directional antennas and facilitates the implementation of such system for road workers, who simply need to place the speed tag at the roadside with a pre-marked side pointing to the traffic flow direction on the carriageway that they want the speed limit to be broadcasted to.

\subsection{Demonstration system}

We have developed a test system with Jennic JN5139 wireless controller and bearing measurement module to demonstrate the concept of our design. The JN5139 microcontroller queries and reads measurement modules via a standard $\mathrm{I} 2 \mathrm{C}$ bus. In our test system the tags transmits a 20 mph speed limit. The condition for judging the validity of received speed limit is $(V H-x)<C B<(V H+x)$. On the screen of the receiver we can consistently display the received speed limit and its $\mathrm{CB}$ value. The receiver then uses above judge condition to compare the $\mathrm{CB}$ value with its own $\mathrm{VH}$ value and determine whether the speed limit received is valid. For simulation purpose the system of the receiver also retrieves the vehicle's actual speed from a separate GPS module so that it could decide whether the vehicle is speeding or not. We test the system with various values for the threshold $\mathrm{x}$ and $x=20$ seemed to be a sweet point where opposite carriage ways and cross roads can be well identified, while at the same the system was not too sensitive to the measurement module bearing error and slight vehicle heading shift on road.

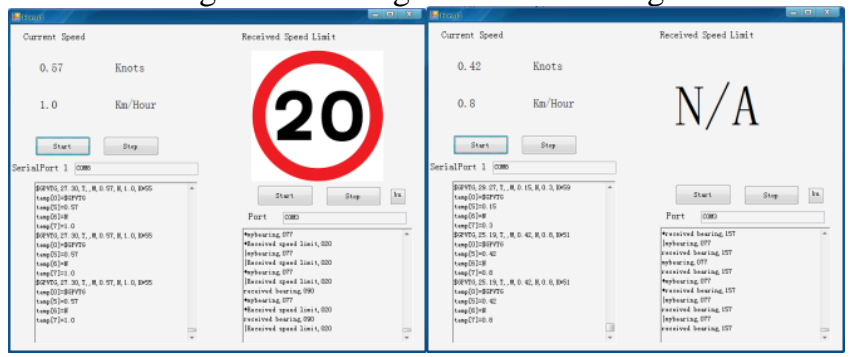

Fig.11. Receiver screen in demonstration system

\section{DISCUSSION AND CONCLUSION}

In this paper we investigated automatic speed limit transmission based on RFID technologies. Both passive and active RFID systems show potential in such application. A Passive RFID system features cheap yet reliable tag design which makes it suitable for massive implementation for large scale applications. Using passive RFID system also brings the benefit of easy maintenance as passive tags do not need battery and can be re-programmed remotely. We have discussed and concluded that passive tag confusion/ghost reading and reader conflicts can be avoided by placing passive tags on road surface.
However, the high cost, bulky size and high energy consumption of current passive readers prevent them from being used for small scale applications such as temporary speed limit in road work or congestion areas. For such scenarios the active RFID is preferred because of their low cost driver side device and potential to be integrated with existing in vehicle systems. They also have a longer read range and more reliable tag reading. The tag confusion issue in active RFID is addressed by introducing and integrating bearing measurement module with tags and readers/receivers. A test system was developed and its performance has demonstrated the feasibility of our design concept.

Future work includes making proper casing for our system to be further evaluated in long-term tests as well as in extreme weather conditions. A more comprehensive driver assistant system will be studied with the current design concept to be combined.

\section{ACKNOWLEDGEMENT}

This research is funded by the United Kingdom East Midlands Development Agency (EMDA) / Transport iNET.

\section{REFERENCES}

Butani, P., John, J., Dhole, A., (2007), Automatic Vehicle Identification Using RFID a First Hand Experience, Indian Railway Technical Bulletin(IRTB), Vol.LXIII No.322.

Cauzard, J. et al., (2004), European drivers and road risk; Part 1: report on principal results. INRETS, Paris

Department for Transport, (2000), New Directions in Speed Management: A Review of Policy.

Kloeden, C. N., Ponte, G. \& McLean, A. J. (2001). Travelling speed and the risk of crash involvement on rural roads. Report CR 204. Australian Transport Safety Bureau ATSB, Civic Square, ACT.

Kohashi, Y., Ishikawa, N., Nakajima,M. (2002), Automatic Recognition of Road signs and Traffic signs, Proc. 1st ITS Symposium 2002, pp.321-326.

Makanae, K., Kanno, A., (2002), Proposal of the Signing System Utilizing Image Recognitio, Proc. 1st ITS Symposium 2002, pp.137-142.

National Highway Traffic Safety Administration. (2009). Traffic safety facts, 2008: speeding. Washington, DC: US Department of Transportation.

Ramakrishnan, K., (2006), Performance benchmarks for passive UHF RFID tags, Proc.13th GI/ITG Conf.Meas., Model. Eval. Comput. Commun. Syst., pp. 137-154.

Yoshimichi, S., and Makanae, K., (2006), Development and Evaluation of In-vehicle Signing System Utilizing RFID tags as Digital Traffic Signs, International Journal of ITS Research, Vol4, No.1, pp.53-58. 